\title{
AVALIAÇÃO REPRODUTIVA DE TOUROS BRANGUS NOS ANOS DE 2010 E 2011
}

\author{
Josimar Zorzo1', Tania Conte², João Luiz Androukovitch¹, Marcio Quint Bugallo \\ 1 CESCAGE \\ 2 UBC - Universidade de British Columbia \\ Correspondência: Josimar Zorzo: josimar.zorzo@hotmail.com
}

\begin{abstract}
RESUMO: O objetivo deste trabalho foi avaliar, através do exame andrológico, a capacidade e evolução reprodutiva de um rebanho de touros comerciais da raça Brangus destinados à reprodução por monta natural nos anos de 2010 e 2011. Neste trabalho foram avaliados no período de 2 anos, 393 touros desta raça com idades entre 16 e 21 meses (G1) e acima dos 32 meses (G2), através de exame andrológico completo. As medidas de circunferência escrotal (CE) do grupo etário G1 apresentou aumento significativo de 2010 para 2011, enquanto que no G2, não se observou diferença entre os períodos. Os animais apresentaram uma boa evolução reprodutiva, pois os índices de reprovação no exame andrológico observados em 2010 foram de $37 \%$ e $22 \%$ no G1 e G2, e $20 \%$ e $27 \%$ respectivamente em 2011 , mas essa diferença entre os períodos não foi significante estatisticamente, permanecendo dentro da faixa de boa atividade reprodutiva. Ao término deste trabalho, observou-se o quanto é importante a realização de exames andrológicos periódicos, que possibilita identificar machos, cujos índices reprodutivos encontravam-se abaixo do preconizado como ideais para a espécie, e exclusão desses animais do programa de monta natural ou comercialização. Isso possibilita melhores resultados de fertilidade do rebanho e o melhoramento genético dos animais, reduzindo os riscos de prejuízos à atividade reprodutiva.
\end{abstract}

Palavras-chave: andrológico; circunferência escrotal; patologia espermática

\section{REPRODUCTIVE EVALUATION OF BRANGUS BULLS IN 2010-2011}

\begin{abstract}
This study aimed to evaluate through andrological examination a herd's reproductive capacity and evolution. 393 bulls from the commercial breed Brangus, assigned for natural breeding in 2010 and 2011 were assessed in both years. The animals aged between 16 and 21 months (G1) and over 32 months (G2) underwent comprehensive andrological examination. The animals in G1 showed a significant increase in scrotal circumference (SC) in 2011 whether this change was not observed in the bulls from G2. The failure rates accounted for $37 \%$ and $22 \%$ in 2010 for $\mathrm{G} 1$ and G2, respectively. In 2011 these reported rates were $20 \%$ and $27 \%$. However, the changes were not statically significant and the rates remained in the range of good reproductive function. We were able to observe the importance of regular andrological examinations. It enable us to identified animals who do not meet optimal reproductive standards and exclude them natural breeding programs or commercialization. Likely, this allows increased fertility and genetic improvement in cattle, which will lower risks of losses in commercial reproductive activity.
\end{abstract}

Key Words: andrological examination; scrotal circumference; spermatic pathology 


\section{INTRODUÇÃO}

$\mathrm{Na}$ utilização de touros em monta natural durante um período de acasalamento é fundamental a avaliação andrológica, para que haja um melhor desempenho reprodutivo. A eficiência reprodutiva de um touro é uma das mais importantes características do rebanho de corte, principalmente em criações extensivas, na qual a reprodução constitui um fator limitante à produção. Para que se possa avaliar a aptidão desses animais destinados à monta natural, o exame andrológico torna-se fundamental e amplamente utilizado, seja em propriedades particulares ou centrais de coletas (Freneau et al., 2006, Addad et al., 2009).

Embora sendo de extrema importância, os exames de sêmen de animais criados a campo e destinados à cobertura por monta natural nem sempre são solicitados pelos pecuaristas. Portanto, uma investigação do histórico, o exame clínico geral da colheita do sêmen e o andrológico completo, oferecendo subsídios seguros para um prognóstico sobre o potencial reprodutivo do animal, é muito importante (Silva et al., 2003, Rossi et al., 2009).

O exame andrológico é uma das técnicas mais utilizadas pelos médicos veterinários para avaliar a fertilidade. Ele caracteriza-se por exame clínico, medida de circunferência escrotal (CE) e avaliação do sêmen para motilidade, vigor, turbilhonamento, concentração e morfologia (Folhadella et al., 2006).

A CE é uma mensuração de fácil obtenção, que possui uma característica de herdabilidade moderada a alta, correlacionada com o ganho de peso, devido à produção de hormônios, produção de espermatozóides e qualidade seminal dos touros.

Testículos com tamanhos iguais podem produzir sêmen tanto de baixa como de alta motilidade, devendo-se a produção de sêmen à funcionalidade testicular do animal e não somente à medida da CE (Silva et al., 2002; Gandolfo, 2007).

Em estudos realizados por Brito et al. (2002), concluiu-se que a produção de espermatozóides foi associada com o volume testicular, circunferência escrotal, cobertura de gordura, e temperatura de superfície escrotal, enquanto a qualidade do sêmen foi associada com a temperatura de superfície escrotal, circunferência escrotal e a forma escrotal.

A seleção de touros com maior $\mathrm{CE}$ e características de crescimento, também vai afetar positivamente a fertilidade das fêmeas, melhorando a eficiência reprodutiva das mesmas (Kriese et al., 1991; Gandolfo, 2007).

Além da avaliação da CE, o exame andrológico avalia a qualidade do sêmen através das características físico-químicas que caracterizam a funcionalidade testicular, sendo eles: aspecto, volume e turbilhonamento; e características microscópicas como motilidade e vigor. Como avaliação complementar, realiza-se a avaliação das patologias espermáticas, classificadas em defeitos maiores, menores e totais, sendo aceitáveis os defeitos maiores de até $20 \%$, e menores de até $10 \%$ totalizando um máximo de até $30 \%$ de defeitos, segundo o manual da CBRA (1998). A morfologia espermática é o parâmetro que o médico veterinário tem como principal marcador para acompanhar a precocidade sexual dos animais (Freneau, 2011).

O objetivo deste trabalho foi avaliar, através do exame andrológico, a capacidade reprodutiva de touros comerciais da raça Brangus destinados à reprodução por monta natural. 


\section{MATERIAL E MÉTODOS}

Foram avaliados 393 touros Brangus, com idades entre 16 e 21 meses $(n=180)$ e acima 32 meses $(n=213)$, através de exame andrológico completo que é constituído de avaliação física e morfológica do sêmen e biometria testicular. Os mesmos eram pertencentes à Companhia Agroflorestal Guapiara, localizados nos municípios de Castro, Laranjal, Teixeira Soares e Boaventura de São Roque situados no estado do Paraná e Ribeirão Branco, no estado de São Paulo. As avaliações andrológicas ocorreram nos meses de abril a junho do ano de 2010 e 2011.

Os animais foram agrupados em duas categorias: Grupo 1 (G1) - animais de 16 a 21 meses; Grupo 2 (G2) animais acima de 32 meses (em trabalho reprodutivo), com o objetivo de avaliar o desenvolvimento testicular, maturidade sexual e aptidão reprodutiva dos mesmos.

Os animais jovens (G1) encontravam-se em um sistema a pasto consorciado de milheto (Pennisetum glaucum) e papuã (Brachiaria plantaginea), com suplemento mineral ad libitum. Os animais jovens recebiam ainda, complemento alimentar a base de farelo de soja, quirera de milho e suplemento vitamínico-mineral. Os animais mais velhos, (G2), encontravam-se exclusivamente em sistema de campo com fornecimento de sal mineral e já haviam sido utilizados na estação reprodutiva.

As medições de Circunferência Escrotal (CE) foram obtidas com auxílio de fita métrica milimetrada, posicionada na região mediana do escroto, no ponto de maior dimensão, envolvendo as duas gônadas e a pele escrotal. As mensurações em centímetros do comprimento e largura testicular foram obtidas com o auxilio da mesma fita métrica. Todas as medidas foram realizadas por um mesmo avaliador. $\begin{array}{ccr}\text { Para a obtenção do sêmen } \\ \text { adotou-se } & 0 \text { método do }\end{array}$ eletroejaculação, sendo os ejaculados coletados em tubos coletores graduados, acoplados a funis plásticos.

Uma vez coletado, foram imediatamente avaliados 0 turbilhonamento, motilidade espermática progressiva (MIP) e o vigor espermático.

O turbilhonamento foi avaliado com auxilio de um microscópio binocular, em aumento de 100x. Para isso, uma gota de sêmen foi colocada sobre uma lâmina previamente aquecida a $37^{\circ} \mathrm{C}$ e classificada de acordo com 0 movimento espermático, em escala de 0 a 5 .

Posteriormente, utilizando a mesma gota de sêmen sobre a lâmina, foram observados, sob aumento de 400x, a motilidade espermática e 0 vigor, sendo classificados em porcentagem (\%) e escala de 0 a 5 , respectivamente.

Para a avaliação das características morfológicas dos espermatozóides, uma alíquota de sêmen de cada ejaculado foi posto sobre uma lâmina e corado com vermelho-congo, sendo avaliadas e contabilizadas, por imersão, 200 células por ejaculado. As anomalias espermáticas foram computadas de acordo com a quantidade de defeitos maiores, defeitos menores e defeitos totais.

Foi determinado por consenso entre os médicos veterinários autores deste trabalho, que os índices de reprovação no primeiro exame seriam considerados ótimos até $20 \%$, bons entre $21-30 \%$, regulares entre $31-40 \%$ e ruins acima de $50 \%$. Os índices de descarte, a partir do reteste, teriam como parâmetros de até $8 \%$ ótimo, 7 $15 \%$ bom, $16-24 \%$ regulares e acima de $25 \%$ ruim.

Para avaliar a alteração das medidas de $\mathrm{CE}$ e funcionalidade testicular dos animais entre os períodos, 
foi utilizado o teste estatístico t-test. Para avaliar as diferentes proporções de animais inaptos e quantidade de descartes entre os períodos foi aplicado o Teste Quiquadrado de Pearson com correção de continuidade de Yates. Finalmente foi aplicado o Teste Exato de Fisher, para avaliação das patologias que motivaram os descartes em cada grupo nos dois períodos.

\section{RESULTADOS E DISCUSSÃO}

Com relação aos resultados obtidos nas médias de CE, observou-se um aumento de $36,78 \mathrm{~cm}$ para $38,18 \mathrm{~cm}$ entre todos os animais de 2010 para 2011. Na análise de subgrupo observamos que essa diferença ocorreu pelo aumento de CE dos animais do G1 de um ano para o outro. Os animais do G2 não apresentaram alteração de CE média entre os anos de 2010 e 2011. (Tabela 1). Com relação ao vigor, em geral observamos uma melhora significativa de 2010 para 2011. O que pode ser atribuído a uma melhora desse parâmetro no G2 em 2011 (Tabela 1). A motilidade espermática e o turbilhonamento também apresentaram melhora significativa no ano de 2011, dessa vez atribuídos a melhora dos parâmetros apresentados pelos animais do G1.

Tabela 1 - Médias e Desvio Padrão dos animais aptos ao Exame Andrológico.

\begin{tabular}{|c|c|c|c|c|c|c|c|c|c|}
\hline & \multicolumn{2}{|c|}{ G1 } & & \multicolumn{2}{|c|}{ G2 } & & \multicolumn{2}{|c|}{$\begin{array}{l}\text { Total } \\
\end{array}$} & \\
\hline & 2010 & 2011 & $p^{2}$ & 2010 & 2011 & $p^{2}$ & 2010 & 2011 & $p^{2}$ \\
\hline Nanimais & 61 & 63 & & 80 & 81 & & 141 & 144 & \\
\hline$\overline{C E}(\mathrm{~cm})^{\prime}$ & $34,67 \pm 2,32$ & $37,19 \pm 3,12$ & $60.001 "$ & $38,39 \pm 246$ & $38,95 \pm 2,63$ & $0.163 t$ & $36,78 \pm 3,03$ & $338,18 \pm 2,98$ & $0.001^{\prime \prime}$ \\
\hline Vigor(15) & $4,12 \pm 0,69$ & $3,93 \pm 0,67$ & $0.145 t$ & $3,9 \pm 0,44$ & $4,48 \pm 0,69$ & $80.001 "$ & $3,99 \pm 0,57$ & $4,24 \pm 0,73$ & $0.001^{\prime \prime}$ \\
\hline Motilidade(Y\%) & $86 \pm 5,46$ & $88 \pm 3,83$ & $0.003^{* x}$ & $87 \pm 4,57$ & $88 \pm 4,08$ & $0.391 t$ & $87 \pm 5,02$ & $88 \pm 3,96$ & $0.008^{\mathrm{r}}$ \\
\hline Tutilhão(1.5) & $271 \pm 1,10$ & $3,25 \pm 1,05$ & $0.005^{* *}$ & $3,4 \pm 0,85$ & $3,46 \pm 1,14$ & $0.721 \dagger$ & $3,1 \pm 1,02$ & $3,37 \pm 1,10$ & $0.034^{\pi t}$ \\
\hline
\end{tabular}

Kastelic et al. (2001), trabalhando com touros de corte provenientes dos cruzamentos com Angus de idade média de 16 meses encontraram em seus estudos uma média de CE de 33,7 $\pm 0,3 \mathrm{~cm}$, valores estes semelhantes aos encontrados neste estudo, no ano de
2010, mas bastante inferior aos observados no ano seguinte. Os resultados também foram semelhantes aos observados por Moraes et al. (1998) e Chacur et al. (2007), analisando touros Brangus-lbagé e Aberdeen Angus com três anos de idade, e corroborados pelos encontrados em nossos estudos. Touros que apresentam maior CE possuem maior característica de ganho de peso e capacidade reprodutiva. Portanto, nosso estudo demonstrou que houve uma melhora no parâmetro de CE entre animais jovens na coorte de 2011 possibilitando uma melhora da capacidade reprodutiva desses animais.

As médias de vigor dos animais adultos, aptos à reprodução, avaliados neste estudo, foram semelhantes em 2010 aos encontrados por Moraes et al. (1998), quando avaliaram touros Hereford e Braford, que encontrara, valores de 3,94 $\pm 0,01$ e superiores no ano seguinte. Nossa amostra foi superior nos parâmetros de motilidade quando comparado às medidas encontradas por esse mesmo pesquisador $(67,17 \% \pm 1,44)$ nos dois períodos. Um sêmen com melhor motilidade e vigor possui melhor capacidade de fecundação no trato reprodutivo da fêmea. Os resultados de turbilhonamento foram bastante superiores quando comparado aos resultados de Chacur et al. (2007), que

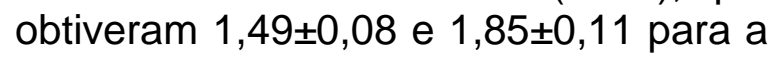
mesma raça e Pardo-Suíço respectivamente.

Dos 97 animais do G1 em 2010, o índice de reprovação no primeiro exame andrológico foi de 37\%, destes, apenas $12,4 \%$ foram descartados após uma segunda avaliação (Tabela 2).

Estes apresentaram CE abaixo de $30 \mathrm{~cm}$, alterações testiculares graves ao exame clínico (vesiculite, epididimite e fibrose epididimária), defeitos de peça reprodutiva (micro pênis) e defeitos espermáticos totais acima de $30 \%$ 
(Tabela 3). Já em 2011, para os 83 animais, o índice de reprovação caiu para $20 \%$, sendo $9,6 \%$ descartados. Os animais jovens que apresentaram azoospermia, ejaculado insuficiente e alterações testiculares leves (epididimite) no momento do exame foram reservados e submetidos a uma nova avaliação andrológica sessenta dias após o exame. Segundo Fonseca et al. (1991), na fase de amadurecimento sexual ocorrem alterações na espermatogênese, o que pode explicar 0 alto percentual de anomalias no mesmo. Apesar da redução de inaptos e descarte em 2011 neste grupo, não se encontrou significância estatística entre os anos nesta diferença.

No G2 em 2010, o índice de reprovação foi de $22 \%$, e $12,7 \%$ foram descartados (Tabela 2). Os 9,3\% temporariamente inaptos, apresentavam algum tipo de alteração espermática ou haviam sido tratados com medicamentos (corticosteróides), o que não foi novamente observado em nova avaliação após dois meses. No ano de 2011, o índice de inaptidão foi de $27 \%$, e $17,1 \%$ de descarte. Estes números se elevaram no segundo período de avaliação, no entanto, essa diferença entre os anos também não foi estatisticamente significativa. Atribuímos esse aumento à existência de animais com idade avançada, mas o principal fator foi com relação às patologias espermáticas neste grupo (Tabela 3).

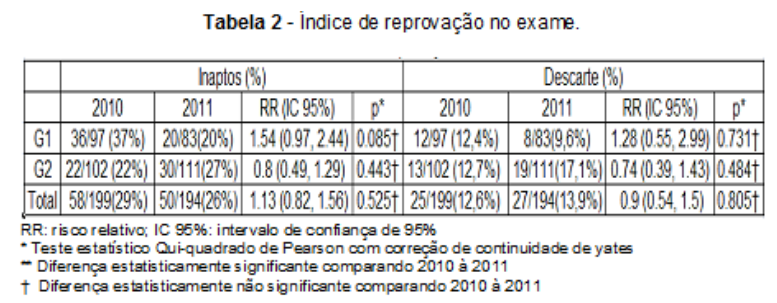

Com relação ao espermograma, os defeitos foram agrupados em maiores, menores e totais, sendo estes, formados pelos resultados de defeitos maiores e menores contados separadamente. Os defeitos maiores têm origem no epitélio seminífero e indicam uma espermatogênese imperfeita (Gandolfo, 2007). Já os defeitos menores, segundo Silva et al. (2003), parte destes são adquiridos durante a passagem pelo ducto deferente ou durante a ejaculação e apresentados por touros com fertilidade normal. Os defeitos foram listados no Gráfico 1.

Segundo Ax et al. (2004), a nutrição, idade, hereditariedade e o ambiente podem interferir na atividade espermática. Alguns animais reprovados em nosso estudo pelo espermograma, apresentavam motilidade, vigor e turbilhonamento aceitáveis, no entanto um grande número de animais apresentavam patologias espermáticas superiores aos índices aceitáveis pela CBRA (1998), que é de até $30 \%$ de defeitos totais.

O aumento no número de animais reprovados por defeitos totais, principalmente no G2, pode estar relacionado à uma possível degeneração testicular leve, que não produz sinais clínicos detectáveis nos testículos e se manifesta quase que exclusivamente pelo aumento da produção de espermatozóides com defeitos, segundo Freneau (2011).
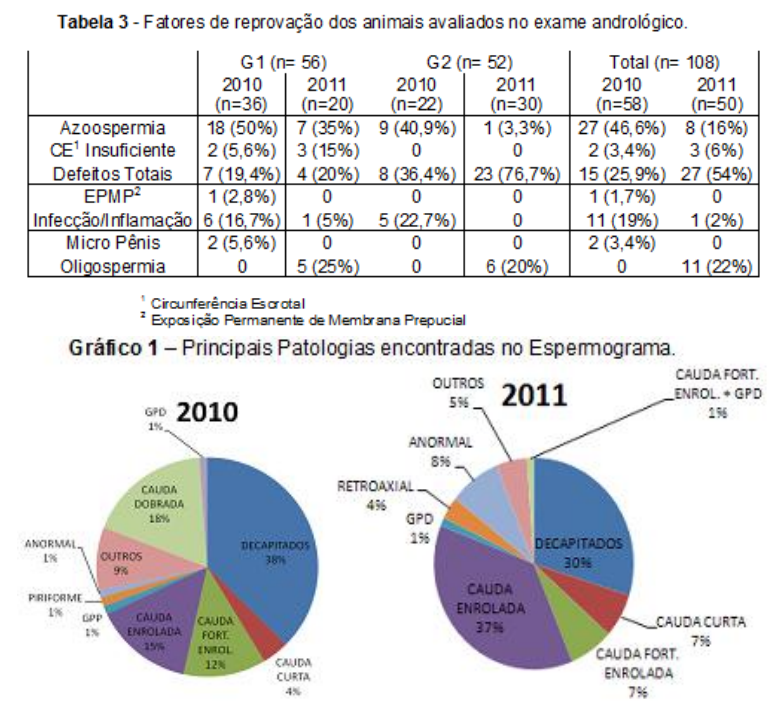
Jainudeen e Hafez (2004) citam bastante a respeito de métodos de identificação da maturidade sexual de animais jovens, no entanto, não há registros de um determinado período em que a idade produtiva começa a interferir na espermatogênese, para que se pudesse determinar a vida útil reprodutiva de um touro.

\section{CONCLUSÃO}

Os animais jovens avaliados no ano de 2011, foram superiores aos de 2010 no que se refere a características espermáticas e circunferência escrotal, demonstrando precocidade e maturidade sexual. Já entre os animais do G2, apesar da melhora nos parâmetros de vigor no corte de 2011, houve um aumento do número de indivíduos adultos que foram reprovados no exame. Apesar desse aumento não ter sido estatisticamente significante, os parâmetros de descarte em 2011 ficaram acima do considerado aceitável para uma boa atividade reprodutiva. Isso pode indicar uma tendência da influência da idade na produção de gametas viáveis.

Avaliando-se a qualidade do sêmen nas suas mais diversas características podemos identificar machos, cujos índices reprodutivos encontravam-se abaixo daqueles preconizados como ideais para a espécie, sendo estes estrategicamente excluídos do programa de monta natural ou mesmo não destinados à comercialização, possibilitando melhores resultados de fertilidade do rebanho e o melhoramento genético dos animais. Com o exame andrológico, pode-se identificar animais com problemas reprodutivos que poderiam estar sendo destinados a monta natural, porém causariam prejuízos a atividade, pois os índices de fertilidade do rebanho seriam baixos.

\section{REFERÊNCIAS}

ADDAD, R. O. et al. Avaliação ClínicoAndrológica em Touros Nelore e Testes de Viabilidade Espermática, Integridade de Acrossoma e Fragmentação de Cromatina ao Longo de três estações reprodutivas. Ciência Animal Brasileira, Goiânia - GO, v.10, n 4, p.1044-1054, out/dez, 2009.

AX, R. L. et al., Avaliação do Sêmen. In: HAFEZ, E. S. E; HAFEZ, B. Reprodução Animal. $7^{\circ}$ ed Barueri - SP: Manole, 2004. Cap. 25, p.369-379.

BRITO, L. F. C. et al. Effect of age and genetic group on characteristics of the scrotum, testes and testicular vascular cones, and on sperm production and semen quality in Al bulls in Brazil. Theriogenology, Canadá, p.1176-1186, 2002.

CBRA. Manual para exame andrológico e avaliação de sêmen animal. $2^{a}$. ed. Belo Horizonte: CBRA, p. 49, 1998.

CHACUR, M. G. M. et al. Relação entre circunferência escrotal, libido, hormônios e características do sêmen em touros Brangus e Pardo-Suíço. Acta Scientiae Veterinariae, Porto Alegre, v.35, n.2, p.173-179, 2007.

FOLHADELLA, I.M. et al. Características andrológicas de touros da raça Gir. Arquivo Brasileiro de Medicina Veterinária e Zootecnia, v.58, n.5, p. 809-815, 2006.

FONSECA, O.F. et al., Procedimentos para Exame Andrológico e Avaliação do Sêmen. Colégio Brasileiro de Reprodução Animal, p.79, 1991.

FRENEAU, G. E. et al. Puberdade em touros Nelore criados em pasto no Brasil:

Características corporais, testiculares e seminais e de índice de capacidade andrológica por pontos. Arquivo Brasileiro de Medicina Veterinária e Zootecnia, Belo Horizonte - MG, v.58, n.6, p.1107-1115, 2006.

FRENEAU, G. E. Aspectos da morfologia espermática em Touros. Revista Brasileira de Reprodução Animal, Belo Horizonte, v.35, n.2, p.160-170, 2011.

GANDOLFO, E. F. Avaliação Reprodutiva em Touros. 2007. 67 f. Dissertação

(Especialização) - Universidade Castelo Branco, São José do Rio Preto, 2007.

JAINUDEEN, M. R.; HAFEZ, E. S. E. Bovinos e Bubalinos. In: HAFEZ, B.; HAFEZ, E. S. E. Reprodução Animal. 7. ed. Barueri: Manole, 2004. Cap.11, p.159-171. 
KASTELIC, J.P.; COOK, R.B.; PIERSON, R.A. et al. Relationships among scrotal and testicular characteristics, sperm production, and seminal quality in 129 beef bulls. Canadian Journal Veterinary Research, v.65, n.2, p.111-115, 2001.

KRIESE, L. A.; BERTRAND, J. K.; BENYSHEK, L. L. Age adjustment factors, heritabilities and genetic correlations for scrotal circumference and related growth traits in Hereford and Brangus bulls. Journal of Animal Science, v.69, n.2, p.478-489, 1991.

MORAES, J. C. F.; HORN, M. M.; ROSADO, JR., A. G. Exame andrológico em touros: qualidade dos indicadores da aptidão reprodutiva em distintos grupos raciais. Ciência Rural, v. 28, n.4, p.647-652, 1998.

ROSSI, R.O.D.S. et al. Características andrológicas e do sêmen de touros do composto Red Norte (Nelore x Tabapuã x Red Angus $x$ Sinepol). Arquivo Brasileiro de Medicina Veterinária e Zootecnia, v.61, n.6, p.1297-1301 2009.

SILVA, A. E. D. F. et al. Fatores Ambientais e Parâmetros Genéticos para Características Produtivas e Reprodutivas em um Rebanho Nelore no Estado do Mato Grosso do Sul. Revista Brasileira de Zootecnia, Viçosa- MG, v.31, n.3, p.1157-1165, 2002.

SILVA, A. E. D. F. et al. Conteúdo de peptídeos e avaliação morfofisiológica dos espermatozóides do epidídimo e ejaculado de bovinos. Revista Brasileira de Zootecnia, v.32, n.6, suppl.2, p.1890-1900, 2003. 